\title{
Anabases
}

\section{La condanna del prologo diegetico euripideo dagli scoli antichi ai trattati del Cinquecento}

La condamnation des prologues diégétiques d'Euripide: des scholies anciennes aux traités du Cinquecento

The Condemnation of Euripides' Diegetic Prologue: from Ancient Scholia to Cinquecento Treatises

\section{Marco Duranti}

\section{OpenEdition}

\section{Journals}

Edizione digitale

URL: https://journals.openedition.org/anabases/8762

DOI: 10.4000/anabases.8762

ISSN: 2256-9421

\section{Editore}

E.R.A.S.M.E.

\section{Edizione cartacea}

Data di pubblicazione: 14 avril 2019

Paginazione: 135-148

ISSN: 1774-4296

\section{Notizia bibliografica digitale}

Marco Duranti, «La condanna del prologo diegetico euripideo dagli scoli antichi ai trattati del Cinquecento», Anabases [Online], 29 | 2019, Messo online il 14 avril 2021, consultato il 06 novembre 2021. URL: http://journals.openedition.org/anabases/8762 ; DOI: https://doi.org/10.4000/anabases 8762 

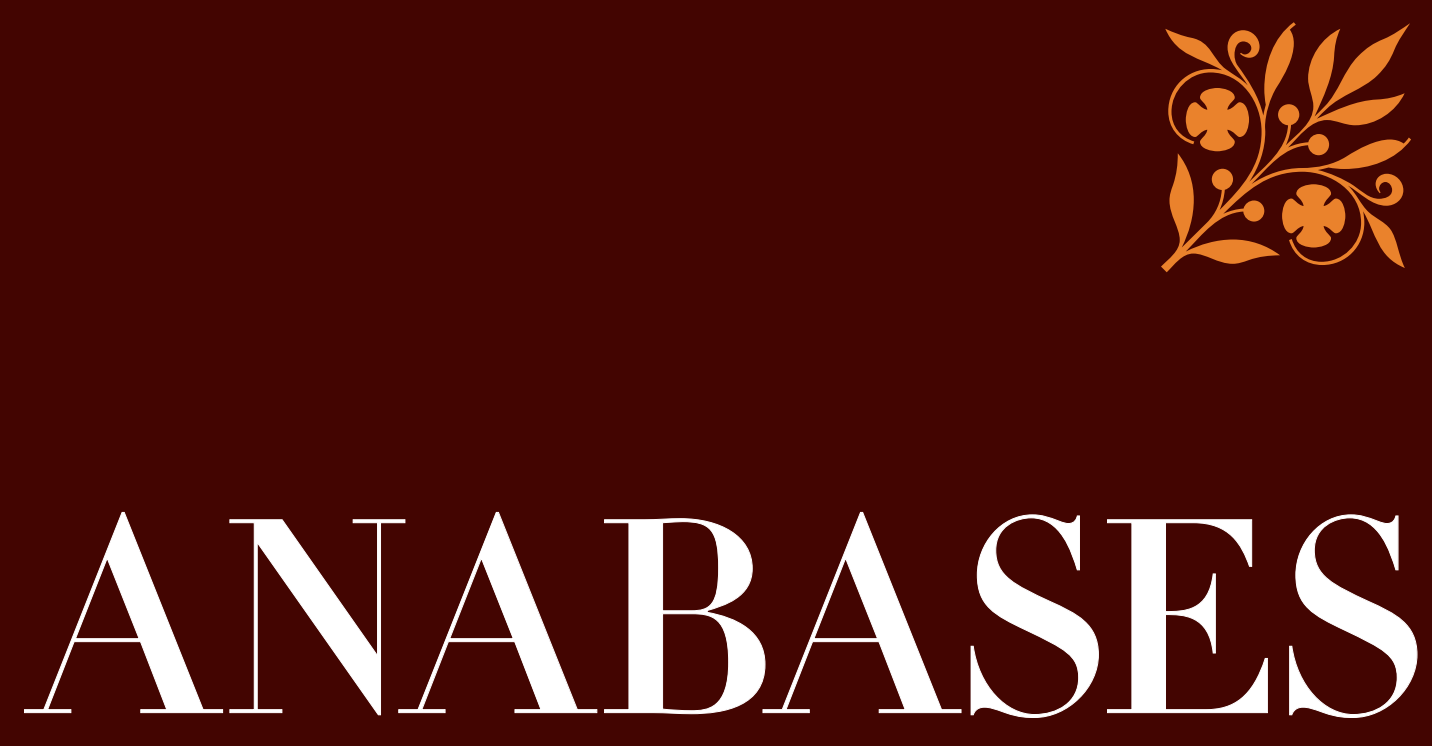

Traditions et Réceptions de l'Antiquité

\section{No29 \\ 2019}

Juliette Ernst Sculptures onctueuses de Meekyoung Shin Théâtre antique et travaux savants dans la Première modernité L'Antiquité dans la peinture (17911880) Réception d'Ovide Pierre Grimal 
ANABASES

Traditions et Réceptions de l'Antiquité

Revue de l'équipe de recherche E.R.A.S.M.E.

Université Toulouse-Jean Jaurès (UT2J)

Anabases dispose d'un Comité de lecture international. Chaque article envoyé à la rédaction est soumis, une fois anonymisé, à l'expertise de deux spécialistes qui rendent un rapport écrit. Les deux rapports anonymisés sont transmis à l'auteur qui tient compte des observations en vue de la publication.

\section{Comité SCIEnTIFIQUe}

Germaine Aujac (université Toulouse-Jean Jaurès : histoire de la géographie et des sciences antiques)

Florence Bouchet (université Toulouse-Jean Jaurès : littérature médiévale)

Hinnerk BruHns (CNRS : histoire économique et sociale ancienne et contemporaine)

Paulo Butti de Lima (université de Bari : historiographie et réception de l'Antiquité)

Luciano CANFora (université de Bari : littérature et histoire anciennes, historiographie)

Giovanna Ceserani (Stanford University : histoire intellectuelle et historiographie de la tradition classique)

Temístocles Cezar (université de Porto Alegre : historiographie moderne)

Serafina Сиомо (University of London, Birkbeck College : histoire des mathématiques et des sciences)

Paul Demont (université de Paris Sorbonne : philologie grecque et héritage classique)

Marie-Laurence Desclos (université de Grenoble II : philosophie de l'Antiquité)

Olivier Devillers (université de Bordeaux 3 - Michel-de-Montaigne : littérature et historiographie latines)

Andrea Giardina (Istituto italiano di scienze umane : histoire du monde romain et de ses réceptions)

Ève Gran-Aymerich (aibl : histoire de l'archéologie et des transferts culturels)

François HaRTog (EHEss : historiographie ancienne et moderne)

Geneviève Hoffmann (université de Picardie : histoire des mondes grecs)

Christian JACOB (CNRS/EHEss : histoire comparée et épistémologie des savoirs)

Suzanne Marchand (Louisiana State University : histoire du classicisme et de l'orientalisme)

Wilfried Nippel (Humboldt Universität Berlin : histoire et historiographie de l'Antiquité)

Sylvie Pittia (université de Paris I-Panthéon Sorbonne : histoire et historiographie du monde romain)

Stéphane Ratri (université de Franche-Comté - Besançon : philologie et héritage latin)

Comité de RÉdaction

Jacques Alexandropoulos, Marielle de Béchillon, Corinne Bonnet, Laurent Bricault, Clément Bur,

Philippe Foro, Adeline Grand-Clément, Anne-Hélène Klinger-Dollé, Véronique Krings,

Thibaud Lanfranchi, Claudine Leduc, Pascal Payen, Grégory Reimond, Catherine Valenti

Éditeur RESPonsable

Pascal PAYen

Université Toulouse-Jean Jaurès (UT2J)

SECRÉTARIAT DE RÉDACTION

Anthony Andurand / Clément Bertau-Courbières / Corinne Bonnet / Clément Bur /

Adeline Grand-Clément / Anne-Hélène Kuinger-Dollé / Véronique Krings /

Catherine Valenti (université Toulouse-Jean Jaurès) / Noémie VillacÈQue (université de Reims)

Sites Web

http://plh.univ-tlse2.fr

Revues.org : http://anabases.revues.org

Aвonnement et vente aU numéro

Éditions De Boccard - 4, rue de Lanneau - 75005 Paris

info@deboccard.com - www.deboccard.com

Tél. : 0033/(0)143260037 - Fax : 0033/(0)143548583 




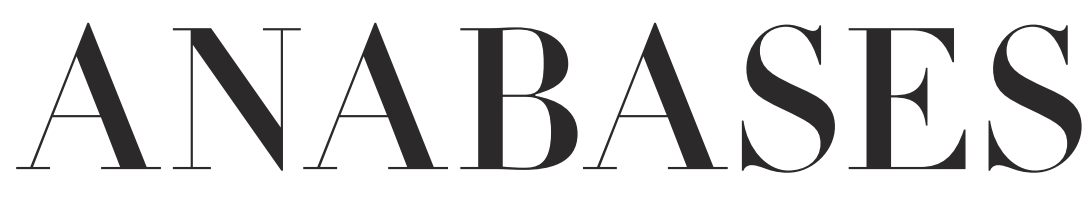

Traditions et Réceptions de l'Antiquité

$$
\begin{aligned}
& N \circ 29 \\
& 2019
\end{aligned}
$$

\section{E.R.A.S.M.E.}

Université Toulouse - Jean Jaurès 



\section{Sommaire}

$\mathrm{N}^{\circ} 29-2019$

\section{Historiographie et identités culturelles}

Ilse Hilbold

Les archives d'une bibliographe des sciences de l'Antiquité :

Juliette Ernst et la fabrique des relations internationales . . . . . . . . . I I3

Vivien LONGHI

La crise, une notion politique héritée des Grecs ? . . . . . . . . . . . 2I

Mireille Lacave-Allemand et Michel Lacave,

L’Antiquité dans la peinture en France, I79I-I880 :

une analyse quantitative à travers les Salons et les Prix de Rome . . . . . 37

Tiphaine Besnard

Du Weathering Project aux autoportraits en Venus :

Les sculptures onctueuses et savonneuses de Meekyoung Shin . . . . . 7 I

\section{Traditions du patrimoine antique}

Dossier dirigé par Pascale Paré-Rey et Malika Bastin-Hammou,

“La réception du théâtre antique dans les travaux savants de l’Europe

de la Première modernité »

Malika Bastin-Hammou et Pascale Paré-Rey

“ La réception du théâtre antique dans les travaux savants

de l'Europe de la Première modernité » . . . . . . . . . . . . . . . . 89

Kevin Bovier

Rétablir la métrique de Térence au $\mathrm{XvI}^{\mathrm{e}}$ siècle :

le cas du Iudicium de Glaréan (1540) . . . . . . . . . . . . . . . . . . . . . . 93 
Brice Denoyer

L'héritage de la métrique antique

dans l'alexandrin français au xvie siècle . . . . . . . . . . . . . IO7

Giovanna Di Martino

Vittorio Alfieri's tormented relationship with Aeschylus:

Agamennone between Tradition and Innovation . . . . . . . . . . . . . . . I2I

Marco Duranti

La condanna del prologo diegetico euripideo dagli scoli antichi

ai trattati del Cinquecento . . . . . . . . . . . . . . .

Rosario López Gregoris

L'influence de l' Arte nuevo de hacer comedias de Lope de Vega

dans l'usage des modèles classiques latins en Espagne

pendant le Siècle d'or et le Baroque » . . . . . . . . . . . . . . . . I49

Cressida Ryan

Sophoclean scholarship as a tool

to interpret eighteenth-century England . . . . . . . . . . . . . . г 6 I

Záviš ŠumaN

Axiologie critique de La Mesnardière . . . . . . . . . . . . . . . . . . . . I79

\section{Archéologie des savoirs}

Dossier dirigé par Cristina Noacco

“2000 ans déjà... Aspects de la réception d'Ovide » . . . . . . . . . . . I93

\section{La réception d'Ovide au Moyen Âge}

Jean-Marie Fritz et Cristina NoAcco

Lire Ovide au xiI ${ }^{\mathrm{e}}$ siècle : Arnoul d'Orléans

commentateur des Métamorphoses . . . . . . . . . . . . . . . $\quad{ }_{195}$

Franck Coulson

Le mythe de Pythagore dans le commentaire

“Vulgate » des Métamorphoses . . . . . . . . . . . . . . . . . . . . . . 2I

Marylène Possamaï

Comment éditer l'Ovide moralisé :

le problème de la mise en page du manuscrit Rouen Bm O.4 . . . . . . . 225

Anneliese Pollock Renck

Les Hérö̈des à la fin du Moyen Âge : pour une définition élargie de l'acte traducteur . . . . . . . . . . . . . . . . . . 239 
II. La réception d'Ovide à l'époque moderne

Fátima Díez Platas et Patricia Meilán Jácome

Le poète dans son œuvre. Ovide dans les images des Fasti

et des Tristia entre les Xv et $\mathrm{xvI}^{\mathrm{e}}$ siècles . . . . . . . . . . . . . . . . . 255

Ana Paula Rebelo Correia

Les représentations des Métamorphoses d'Ovide

dans les azulejos portugais. Influence des modèles gravés français . . . . 269

Sarah ReY

Figures d'Orphée au cinéma . . . . . . . . . . . . . . . . . . . 277

\section{Actualités et débats}

Marine LE BAIL

La modernité littéraire serait-elle affaire d'Antiquité(s) ?

Euvres \& Critiques: La contribution de l'archéologie à la genèse

de la littérature moderne, XLII, I, René Sternke dir., 20I7, 338 p. . . . . . . 2 29I

\section{Lire, relire la bibliothèque des sciences de l'Antiquité}

Éric Morvillez

“Les Horti Tauriani de Pierre Grimal

ou les prémices des Jardins romains » . . . . . . . . . . . . . . . . 30I

Pierre Grimal

“Les Horti Tauriani. Étude topographique sur la région

de la Porte Majeur ",MEFRA, tome 53, rg36. p. 25o-286 . . . . . . . . . . . 3 3i3

\section{L'atelier de l'histoire : chantiers historiographiques}

L'Antiquité au musée (coordonné par Adeline Grand-Clément) (6)

Aurélie Rodes, Catherine Valenti

Les Gaulois au musée . . . . . . . . . . . . . . . . . . . .

355

L'Atelier des doctorants (coordonné par Adeline Grand-Clément) (16)

Andrea Avalli

La question étrusque dans l'Italie fasciste $\ldots \ldots \ldots$. . . . . . . 360 
Droit et réception de l'Antiquité

(coordonné par Marielle de Béchillon et Hélène Ménard) (6)

Entre Clio et Thémis. Entretien avec Dario Mantovani, réalisé par

Hélène Ménard (Maître de Conférences d'Histoire romaine, à l'Université

Paul Valéry - Montpellier III), le 22 juin 20I8, à l'occasion de la parution

aux Belles Lettres du livre Les juristes écrivains de la Rome antique.

Les ouvres des juristes comme littérature (juin 20ı8) et de la création

de la chaire “ Droit, culture et société de la Rome antique »

au Collège de France $\left(\mathrm{I}^{\mathrm{er}}\right.$ novembre $\left.20 \mathrm{I} 8\right) \ldots$. . . . . . . . . . . . .

\section{Comptes rendus}

Philippe Borgeaud et Sara Petrella

Le singe de l'autre.

Du sauvage américain à l'histoire comparée des religions (A. Guedon) . . $\quad 37 \mathrm{I}$

Roberta Casagrande-Kim, Samuel Thrope et Raquel Ukeles (éd.)

Romance and reason. Islamic transformations of the classical past

(Cl. Bertau-Courbières) . . . . . . . . . . . . . . . . . . . . .

Hinnerk BruHns

Max Webers historische Sozialökonomie.

L'économie de Max Weber entre histoire et sociologie (Th. Lanfranchi) . . 374

Andrea Cozzo

Riso e sorriso, e altre saggi sulla nonviolenza nella Grecia antica,

(Fr. Pr. Barone) . . . . . . . . . . . . . . . . . . . 377

Franz Cumont

Manichéisme (St. Ratti) . . . . . . . . . . . . . . . 378

Emmanuelle HÉnin et Valérie NAAs (dir.)

Le mythe de l'art antique (Cl. Evrard) . . . . . . . . . . . . . . . . . 380

Jacques Jouanna, Henri Lavagne, Alain Pasquier,

Véronique SchiLtz et Michel Zink (éd.)

Au-delà du Savoir : Les Reinach et le Monde des Arts (G. Hoffmann) . . . .

382

Mario Liverani

Imagining Babylon: The Modern Story of an Ancient City (C.Bonnet) . . . 386

Françoise-Hélène Massa-Pairault, Claude Pouzadoux (Dir.)

Géants et Gigantomachie entre Orient et Occident (C.Giovénal) . . . . . . .

Scott McGill, Joseph Pucci (éd.)

Classics renewed. Reception and Innovation in the Latin Poetry

ofLate Antiquity (S. Clément-Tarantino) . . . . . . . . . . . . . . . . . . . 
Maxwell T. PAule

Canidia, Rome's First Witch (C. Landrea) . . . . . . . . . . . . . . . . . . . 39г

Jessica Priestley, Vasiliki Zali (éd.)

Brill's Companion to the Reception of Herodotus in Antiquity

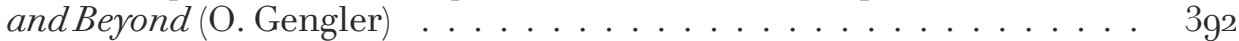

Salvatore QuAsimodo

La Lyre grecque $(\mathrm{M}$. Bianco) . . . . . . . . . . . . . . . . . . 395

Brett M. Rogers, Benjamin Eldon Stevens (éd.)

Classical Traditions in Modern Fantasy (M. Scapin) . . . . . . . . . . . . . 397

Maria Teresa Schettino et Céline UrLacher-Becht (dir.)

Ipse dixit. L'autorité intellectuelle des Anciens : affirmation,

appropriations, détournements (C. Psilakis) . . . . . . . . . . . . . 398

Guy G. Stroumsa

Religions d'Abraham : histoires croisées (D. Lorin) . . . . . . . . . . . . 400

Jean Yvonneau (éd.)

La Muse au long couteau. Critias, de la création littéraire

au terrorisme d'État (G. Hoffmann) . . . . . . . . . . . . . . 405

Résumés . . . . . . . . . . . . . . . . . . . . . 409

Index .............................. 423 



\section{Traditions}

\section{du patrimoine antique}

\section{Dossier dirigé par Pascale Paré-Rey et Malika Bastin-Hammou,}

“ La réception du théâtre antique dans les travaux savants de l'Europe de la Première modernité » 

Anabases 29 (2019), p. 135-148.

\section{La condanna del prologo diegetico euripideo dagli scoli antichi ai trattati del Cinquecento}

Marco Duranti

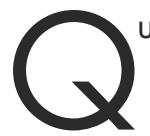

vesto articolo si propone di illustrare un aspetto della ricezione della tragedia antica nel Rinascimento: le riflessioni teoriche su un elemento particolarmente controverso della drammaturgia euripidea, il prologo monologico. ${ }^{1}$ È noto che Euripide introduce regolarmente all'inizio dei suoi drammi un personaggio che, solo in scena, pronuncia un monologo nel quale espone quel complesso di informazioni che i commentatori antichi indicavano con il termine v́лó $\theta \varepsilon \sigma \iota \varsigma$ (di qui in poi traslitterato hypothesis): nella definizione di Rose Meijering, il termine racchiude le risposte a domande quali “chi è in scena?», “dove è ambientato il dramma?», “che cosa è successo prima?». ${ }^{2}$ Questi monologhi

1 Il prologo monologico euripideo è oggetto di una corposa bibliografia, che è qui possibile citare solo in parte. Per un'analisi strutturale si vedano W. Nestre, Die Struktur des Eingangs in der attischen Tragödie, Hildesheim, 1967; H. W. Sснміdт, "Die Struktur des Eingangs", in W. Jens (a cura di), Die Bauformen der griechischen Tragödie, München, 1971, p. 1-46. Per un’analisi narratologica cfr. P. Albini, “Prologo e azione in Euripide», Acmè 40 (1987), p. 31-50; C. SEgal, “Tragic Beginnings: Narration, Voice, and Authority in the Prologues of Greek Drama», YClS 29 (1992), p. 85-112; N. Lowe, “Euripides», in I. J. F. De Jong, R. Nünlist, and A. Bowie (a cura di), Narrators, Narratees, and Narrative in Ancient Greek Literature: Studies in Ancient Greek Narrative, Leiden and Boston, 2004, vol. 1, p. 269-80 (in particolare p. 270-3); I. J F. De Jong, “Sophocles Trachiniae 1-48, Euripidean Prologues, and their Audiences», in R. J. Allan e M. Buiss (a cura di), The Language of Literature: Linguistic Approaches to Classical Texts, Leiden, 2007, p. 7-28.

2 R. Meijering, Literary and Rhetorical Theories in Greek Scholia, Groningen, 1987, p. 117. 
posso però facilmente apparire scarsamente inseriti nel dramma, dal momento che il prologante inizia a parlare senza avere una plausibile ragione per farlo e si produce in un discorso ricco di fatti e particolari che, sebbene abbiano un'utilità informativa per lo spettatore, sono per lo più slegati dalla situazione drammatica in cui il personaggio stesso è calato. Il testo di questi monologhi è inoltre privo di espressioni di pathos e di emotività, cosicché essi appaiono come uno spassionato resoconto fattuale. Tale scollamento del monologo dal contesto drammatico ha indotto molti a pensare che il prologo si collochi prima dell'inizio della tragedia vera e propria e, pur in assenza di esplicite forme allocutive come vocativi o seconde persone, sia rivolto agli spettatori. ${ }^{3}$ I prologhi euripidei violerebbero insomma quel principio che è generalmente osservato in tragedia e che va sotto il nome di verosimiglianza, avvicinandosi in questo al genere della commedia.

L'anomalia del prologo euripideo ha pertanto suscitato e suscita ancora un vivace dibattito nella letteratura critica, che non è qui possibile ripercorrere. ${ }^{4}$ In questo articolo è selezionato un solo, ma cruciale periodo di cimento critico con il prologo euripideo: il Cinquecento. I trattatisti di quest'epoca, alle prese con il compito di elaborare una teoria dei generi drammatici, che avrebbero consegnato alle generazioni future, non potevano non dedicare attenzione a una struttura drammaturgica che sembrava sfidare le regole e i confini tra i generi, e che si inseriva a fatica nelle categorie di analisi che l'antichità stessa aveva elaborato attorno al teatro. Quello che viene affrontato nell'articolo si rivelerà dunque un capitolo tutt'altro che secondario del recupero del dramma antico in età moderna, in quanto esaminando i giudizi formulati sul prologo è possibile ripercorrere il processo di formazione di una teoria moderna del genere tragico, nella dialettica tra aderenza alle fonti antiche e presa di distanza da esse.

3 In epoca più recente, vedono un'allocuzione diretta al pubblico, tra gli altri, M. Pohlenz, La tragedia greca (ed. or. Die griechische Tragödie), Brescia, 1961, vol. I p. 500-1; S. Goldhill, Reading Greek Tragedy, Cambridge, 1986, p. 246; I. J. F. DE Jong, «Sophocles' Trachiniae». Nega invece ogni allocuzione al pubblico nella tragedia D. BAIN, “Audience Address in Greek Tragedy», CQ 25 (1) (1975), p. 13-25.

4 Sulla funzione del prologo nell'economia del dramma è interessante l'opinione di A. W. Verrall, il quale connette il prologo al deus ex machina che caratterizza molti finali euripidei, argomentando che queste due strutture formano una cornice finalizzata a istituire un superficiale collegamento con il mito e con il contesto cultuale in cui veniva messa in scena la tragedia, mentre nella tragedia vera e propria emergeva lo scetticismo di Euripide verso la tradizione (Euripides the Rationalist: A Study in the History of Art and Religion, Cambridge, 1895). Sostanzialmente sulla sua falsariga si collocano N. Terzaghi, "Finali e prologhi euripidei», Dioniso 6 (6) (1938), p. 304-13; M. Pohlenz, La tragedia greca, vol. I p. 499; W. Sснміdт, Der Deus ex Machina bei Euripides, diss. Tübingen, 1963, p. 212-13. 
Proprio al fine di valorizzare il rapporto con le fonti, il primo paragrafo dell'articolo è dedicato agli autori dell'antichità che si occuparono del prologo tragico; il secondo paragrafo illustra poi il lavorio compiuto dai trattatisti rinascimentali su queste basi.

\section{Dalle fonti antiche ai trattati rinascimentali}

Gli studiosi del Rinascimento potevano trovare la definizione del prologo drammatico nella Poetica aristotelica: cil prologo è l'intera parte della tragedia

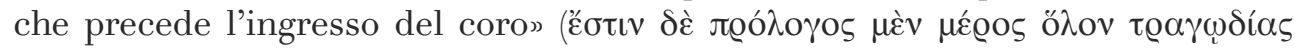

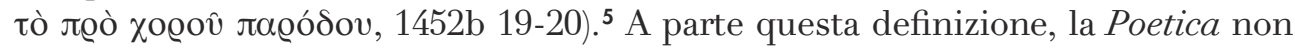
offriva altri elementi di interesse. Erano invece ricchi di spunti gli scoli alla tragedia, che divennero gradualmente disponibili in edizioni a stampa nel corso del Cinquecento. ${ }^{6}$ Essi esprimevano una concorde svalutazione dei prologhi euripidei, ai quali imputavano i seguenti difetti:

- assenza di pathos e di efficacia drammatica (sch. Eur. Tro. 36; sch. Aesch. Eum. 1a);

- allocuzione diretta al pubblico (sch. Eur. Tro. 1; 36);

- eccessiva lunghezza (Vita Eur. 3,14; sch. Eur. Ph. 88; sch. Aristoph. Ach. 416a; sch. Soph. Ai. 38a; ${ }^{7}$ sch. Soph. OC 220);

- prematura rivelazione degli elementi dell'intreccio, con il risultato di pregiudicare l'interesse del pubblico verso il dramma (sch. Hom. Il. 15,64c ${ }^{8}$ ).

5 La Poetica è citata secondo Aristotelis de arte poetica liber, ed. R. Kassel, Oxford, 1965. Tutte le traduzioni dagli autori antichi sono mie.

6 Scoli a Sofocle: 1518 (Commentarii in septem tragedias Sophoclis, ed. Lascaris, Roma); scoli a Euripide: 1534 (Scholia in septem Euripidis tragoedias ex antiquis exemplaribus ab Arsenio archiepiscopo Mombasiae collecta, et nunc primum in lucem edita, Venetiis); scoli a Eschilo: 1552 (Scholia in Aeschyli tragoedias omnes, ed. Robortello, Venetiis); scoli ad Aristofane: 1525 (Aristophanis comoediae novem cum commentarïs antiquis, Florentiae).

7 Lo sch. Ai. 38a non cita espressamente Euripide, ma nota con approvazione che le premesse dell'Aiace vengono rivelate progressivamente ( $x \alpha \tau \grave{\alpha} \beta \varrho \alpha \chi \grave{v})$ attraverso gli

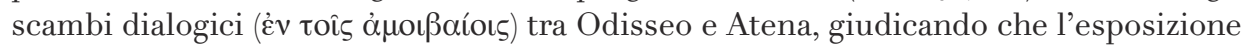
in forma narrativa sarebbe fonte di noia ( $\delta\llcorner\varepsilon \xi \varepsilon \lambda \theta \varepsilon \hat{v}$ ). $\grave{E}$ assai probabile che lo scoliaste abbia in mente il modello negativo dei prologhi euripidei.

8 Lo scoliaste (forse Didimo) registra che Zenodoto espungeva la parte del discorso di Zeus nella quale il dio rivelava la futura uccisione di Patroclo da parte di Ettore, la conseguente vendetta di Achille, la presa di Troia da parte degli Achei (Il. 15,64-77): 
In questo elenco di critiche ci interessano maggiormente i punti 1 e 2 , che sono collegati nello scolio a Tro. 36: qui lo scoliaste biasima Euripide per aver portato in scena un osservatore emotivamente poco coinvolto negli eventi e suggerisce che sarebbe stato meglio introdurre direttamente Ecuba nell'atto di lamentarsi

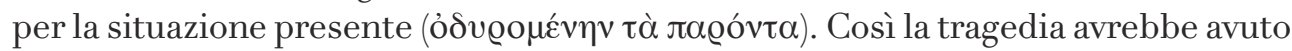

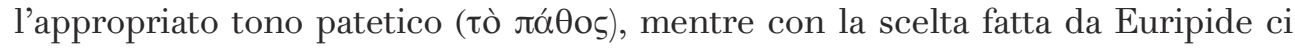

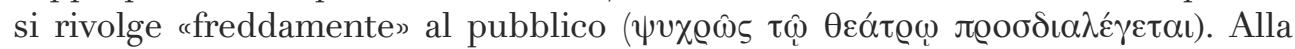
base di questo scolio è una nozione di illusione drammatica, che a sua volta è collegata al concetto di pathos, nella misura in cui, a giudizio del commentatore, solo la creazione di una autentica atmosfera tragica garantisce che lo spettatore venga calato nel dramma e non ne percepisca il carattere artificiale, fino al punto di pensare di essere il locutario della comunicazione del prologante. Su questa linea si pone anche lo sch. Aesch. Eum. 1a, relativo all'entrata in scena della Pizia all'inizio delle Eumenidi: ${ }^{9}$

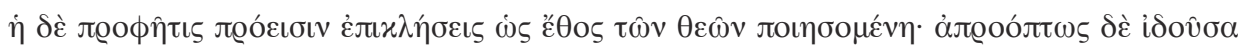

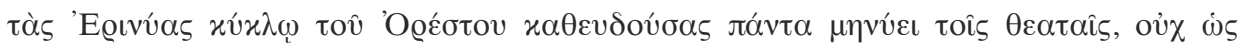

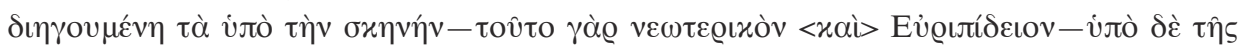

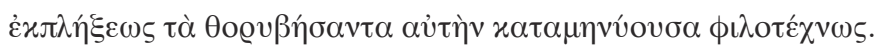

La Pizia si fa avanti per compiere l'invocazione rituale agli dei; dopo aver visto all'improvviso le Erinni che dormono in cerchio attorno a Oreste, comunica tutto agli spettatori: non racconta semplicemente ciò che accade dietro la scena - questa infatti è un'innovazione di Euripide - bensì rivela, in preda allo spavento, ciò che l'ha sconvolta: un espediente artisticamente efficace.

secondo Zenodoto, infatti, questi versi assomigliavano alle inopportune prolessi dei prologhi euripidei (il riferimento è a prologhi marcatamente prolettici come quelli dell'Ippolito o dell'Ione). Lo scoliaste difende tuttavia i versi, argomentando che Omero

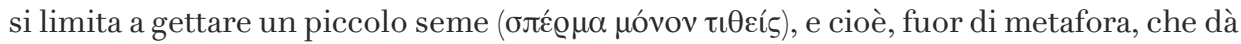
soltanto una parziale anticipazione degli avvenimenti, cosicché non solo non cancella

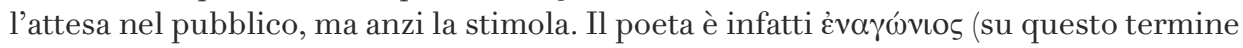
cfr. R. MeiJering, Literary and Rhetorical Theories, p. 205 e nota 212, con relativa bibliografia). Sul concetto di prolessi negli scoli cfr. anche R. NüNLIst, The Ancient Critic at Work, Terms and Concepts of Literary Criticism in Greek Scholia, Cambridge, 2009, p. 34-42.

9 Lo scolio è citato secondo O. L. Sмiтн, (ed.), Scholia Graeca in Aeschylum quae exstant omnia, 1: Scholia in Agamemnonem, Choephoros, Eumenides, Supplices continens, Stuttgart e Leipzig, 1993. Ha ragione R. NüNLIst, The Ancient Critic' p. 342 n. 18, nel ritenere che questo scolio non rimproveri a Euripide di rivolgersi agli spettatori (come invece interpreta R. Meijering, Literary and Rhetorical Theories, p. 195), bensì di presentare a essi un monologo non motivato drammaticamente. 
È chiaro che per lo scoliaste l'esternazione patetica della sacerdotessa eschilea, ben motivata come reazione emotiva alla vista delle Erinni, è da preferirsi alla semplice narrazione dei personaggi prologanti euripidei.

Nell'insistere sull'idea di pathos ${ }^{10}$ e di emozione tragica, gli scoli pongono come elemento centrale di analisi e valutazione di un dramma l'effetto che questo ottiene sullo spettatore, la reazione emotiva che si ingenera in chi assiste allo spettacolo. Anche i rimproveri 3 e 4 a Euripide sono dettati dall'idea che le sue soluzioni drammaturgiche pregiudichino il godimento del dramma da parte dello spettatore: una rhesis prologica troppo lunga causa noia, la rivelazione di troppi elementi dell'intreccio cancella l'interesse ad assistere al dramma fino alla fine. Tutto ciò ci porta a concludere che gli scoli analizzano la poesia drammatica da una prospettiva che possiamo definire retorica: una prospettiva, cioè, che subordina ogni elemento della costruzione del dramma alla necessità di catturare emotivamente lo spettatore, di "persuaderlo».

È questo un punto di grande importanza per noi, in quanto corrisponde al modo in cui i rinascimentali guardavano all'arte poetica. La lettura degli scoli poté perciò riconfermare i trattatisti del Rinascimento in una visione retorica della poesia, in particolare drammatica, che aveva le sue premesse in altre fonti antiche, in specie latine, che più di quelle greche avevano esercitato in modo continuativo la sua influenza dall'età antica al Rinascimento. Come è stato da tempo chiarito, la principale di queste fonti è l'Ars poetica di Orazio, un'opera che, scrive Bernard Weinberg, «considera le forme drammatiche innanzitutto in relazione alla loro capacità di dilettare e di istruire un determinato pubblico, che assisterà ad esse in una determinata epoca e in determinate circostanze" (considers above all the dramatic forms, in relation to their capacity to please and to instruct an audience of a given kind that would see them in a given age under given circumstances). ${ }^{11} \mathrm{In}$ altre parole, l'epistola oraziana insiste sulla necessità che l'opera teatrale risponda alle esigenze dello specifico pubblico che vi assiste affinché possa conquistarne la benevolenza, e con ciò istruirlo. Non meraviglia che i precetti oraziani potessero essere integrati dagli studiosi rinascimentali con ulteriori spunti da fonti, propriamente retoriche, quali Cicerone e Quintiliano.

L'indirizzo retorico dell'analisi poetica, così costituito, condizionò anche la lettura della Poetica aristotelica - che al contrario dell'opera di Orazio entrò solo

10 Sulla necessità che il poeta, l'oratore e persino lo storico suscitino pathos, avvertita in numerose fonti antiche come lo Pseudo longino e Quintiliano, vd. R. Meisering, Literary and Rhetorical Theories, p. 31.

1 B. Weinberg, A History of Literary Criticism in the Italian Renaissance, 2 voll., Chicago, 1961, p. 71. Cfr. B. Kappl, Die Poetik des Aristoteles in der Dichtungstheorie des Cinquecento, Berlin e New York, 2006, p. 15-29. 
gradualmente a far parte del bagaglio critico degli studiosi del Cinquecento con il risultato di travisare le categorie critiche aristoteliche. ${ }^{12}$ Se Aristotele era primariamente interessato alla costruzione dell'intreccio tragico, e perciò valutava l'opera teatrale sulla base di un criterio strutturale, interno all'opera stessa, il suo trattato fu interpretato come se accordasse la preminenza a un criterio esterno di valutazione, incentrato sul pubblico.

Ai nostri fini è importante mettere a fuoco lo slittamento della nozione di verosimiglianza drammatica. In Aristotele, tale concetto è espresso tramite il termine eixós, che indica la congruenza delle azioni con il carattere del personaggio che le compie (vd. ad es. 1451a 38): un intreccio tragico sarà tanto più verosimile quanto più le azioni rappresentate saranno plausibili per il personaggio agente, sulla base della caratterizzazione psicologica che il poeta ne ha fatto. cixós è inoltre utilizzato ad indicare quegli intrecci tragici ben congegnati, nei quali la successione degli eventi segue un rigoroso ordine causale (1452a 20). Si tratta insomma di un concetto strutturale, che pertiene alla costruzione dell'intreccio in coerenza con la costruzione del personaggio. ${ }^{13}$

In un'opera come quella oraziana, caratterizzata da una visione retorica per la quale l'attenzione si sposta dalla costruzione dell'intreccio alla persuasione dell'uditorio, si coglie invece una maggiore insistenza sulla necessità che ciò che è rappresentato aderisca alla realtà, all'esperienza reale del mondo: il poeta romano sentenzia “siano verosimili le cose che si inventano per dare diletto» (v. 338; ficta voluptatis causa sint proxima veris). Se il fine della poesia è - retoricamente - catturare lo spettatore, è necessario che non lo si disorienti con fatti che contraddicono la sua esperienza della realtà; nella poesia drammatica, ciò significa creare un mondo di illusione, secondo un principio di verosimiglianza. Questo criterio, ispirato primariamente da Orazio, ma applicato anche ad Aristotele, si poté dunque armonizzare con le posizioni espresse negli scoli, a formare un quadro teorico coerente all'interno del quale i prologhi euripidei non potevano rientrare. Infatti, poiché i prologanti euripidei parlavano lungamente in assenza di un interlocutore, i loro atti di parola non potevano essere considerati una plausibile mimesi di atti di parola reali. Tale incompatibilità è alla base dei giudizi di condanna dei trattatisti rinascimentali che approfondiremo.

12 Sulla riscoperta della Poetica vd. B. Weinberg, A History, p. 349-423.

13 Sarebbe però un errore ritenere che Aristotele sia disinteressato al coinvolgimento emotivo dello spettatore; questa idea è contraddetta da passi della stessa Poetica (es. 1455a 29-32) e dalla stessa celebre dottrina della catarsi. Il punto è che, per lo Stagirita, la tragedia non deve cercare di suscitare nello spettatore una qualunque forma di emozione, bensì soltanto quella che si ingenera dall'apprezzamento di un intreccio sapiente e bilanciato; con ciò il criterio strutturale conserva la sua centralità (cfr. S. Halliwell, Between Ecstasy and Truth. Interpretation of Greek Poetics from Homer to Longinus, Oxford University Press, 2011, p. 227). 
Ma per continuare con la rassegna delle fonti antiche, è necessario citare un'altra opera, non sempre tenuta nella debita considerazione, che contribuì a consolidare l'accostamento tra poetica e retorica, con importanti riflessi sulla valutazione del prologo: la Retorica aristotelica. Ai nostri fini è rilevante in particolare il passo nel quale Aristotele paragona i prologhi drammatici ai proemi delle orazioni giudiziarie (1415a 8-21): ${ }^{14}$

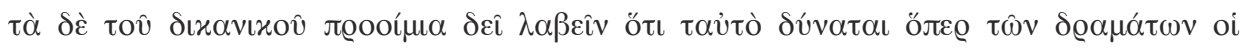

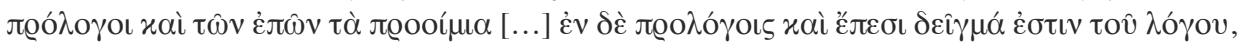

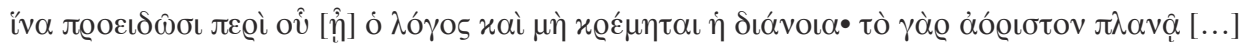

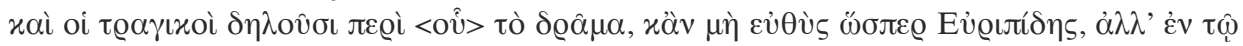

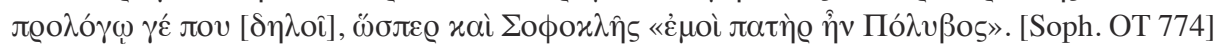

Bisogna comprendere che i proemi dei discorsi giudiziari hanno la stessa funzione che nei drammi hanno i prologhi e nei poemi epici i proemi [...] Nei prologhi e nei poemi epici viene anticipato il soggetto, affinché gli spettatori sappiano su cosa verte il discorso e non rimanga sospeso il pensiero: infatti, ciò che non è ben definito fa smarrire l'ascoltatore. [...] Anche i poeti tragici svelano l'argomento del dramma, anche qualora non lo facciano subito come Euripide, ma comunque da qualche parte nel prologo, come Sofocle: “mio padre era Polibo» [Soph. OT 774].

Secondo Aristotele, l'analogia tra prologhi e proemi giudiziari risiede nella funzione di esporre il soggetto dell'incipiente dramma o orazione; gli elementi, insomma, della hypothesis. Lo stagirita rileva poi una differenza tra Euripide e Sofocle: mentre il primo rivela tutti i presupposti del dramma all'inizio, il secondo fa attendere i suoi spettatori fino a uno stadio successivo del dramma, come dimostra l'esempio dell'Edipo re. Nonostante le difficoltà di interpretazione del testo aristotelico, ${ }^{15}$ risulta chiara la singolarità di Euripide nel rivelare subito gli elementi del dramma; una singolarità che Aristotele si limita a rilevare, senza una

14 La Retorica è citata secondo Aristotle: Rhetoric, vol. 3, ed. by E. M. Cope and J. E. SAndys, Cambridge, 2009 (1877).

15 Il testo tràdito crea difficoltà in questo punto, perché sembra difficile che Aristotele possa considerare il verso 774 della tragedia sofoclea, dove Edipo rivela chi è il suo presunto padre, come facente parte del prologo; si può però intendere che il filosofo usi il termine "prologo» a indicare non solo la scena iniziale della tragedia, ma anche discorsi introduttivi posti in altre parti. In alternativa si potrebbe accettare

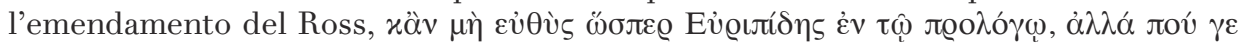
(«anche qualora lo facciano [scil. anche qualora i drammaturghi rivelino i presupposti del dramma] non immediatamente come Euripide nel prologo, ma in qualche altro punto del dramma»), ma indipendentemente dalla sua plausibilità, è più opportuno citare il passo nella forma restituita dai codici, perché in quella forma lo leggevano i trattatisti rinascimentali. 
chiara espressione di preferenza per il modello euripideo o per quello sofocleo. Tutttavia la brachilogia del testo aristotelico poteva facilmente creare ambiguità su questo punto, soprattutto in una resa traduttiva. Così ad esempio nella traduzione di Giorgio Trapezunte ${ }^{16}$ si legge tragici quoque fabulam significant, quamvis non statim, ut Euripides: la formulazione sembra far pendere il testo aristotelico verso un giudizio negativo su Euripide.

A parte questo problema traduttivo, la Retorica aristotelica si inserisce nella nostra trattazione perché, istituendo un'analogia che istituisce tra il prologo e il proemio dell'orazione giudiziaria, induceva a ritenere che anche Aristotele condividesse quell'orientamento retorico che, come abbiamo visto, caratterizzava i critici rinascimentali nell'accostarsi alle opere teatrali, contribuendo così a ispirare una lettura in chiave retorica della Poetica.

Resta da considerare un'ultima fonte antica che riconfermò i rinascimentali nella convinzione che il prologo diegetico fosse inadatto al genere tragico: il breve trattato che va sotto il nome di De Comoedia, tramandato insieme al commento di Donato alle commedie di Terenzio e regolarmente incluso nelle numerose edizioni a stampa di questo autore. ${ }^{17} \mathrm{I}$ due passi per noi più rilevanti sono de com. 4,5, attribuibile a Evanzio, un critico della generazione precedente a quella di Donato; de com. 7,1-4, scritto con ogni probabilità dallo stesso Donato. ${ }^{18} \mathrm{Il}$ primo di questi passi contiene una peculiare divisione delle parti della commedia, di origine peripatetica e forse risalente allo stesso Aristotele, ${ }^{19}$ nella quale il prologo (prologus) è considerato come una prefazione al dramma propriamente detto, nonché l'unica parte del dramma nella quale il drammaturgo è autorizzato a rivolgersi al pubblico: ${ }^{20}$

16 La traduzione di Trapezunte fu pubblicata per la prima volta nel 1478 a Venezia con il titolo di Aristotelis Rhetoricorum libri quinque ex interpretatione Georgii Trapezunti. Ho potuto consultarla nell'edizione del 1541, pubblicata a Lione, intitolata Aristotelis Rhetoricorum ad Teodecten libri tres, Georgio Trapezunte interprete.

17 Sulle edizioni a stampa di questo trattato cfr. G. Capaiuolo, “Antiche edizioni del De fabula di Evanzio", BStudLat 7 (1977), p. 42-51.

18 Si veda A. Primmer, “Akte und Spannung: zur hellenistischen Theorie der Komödienstruktur bei Aelius Donatus», Act. Ant. Hung. 48 (2008), p. $404-32$ (in particolare p. 405).

19 Per A. Rostagni, Donato dipende da Aristotele e Teofrasto, ma tramite fonti intermedie latine, probabilmente Varrone o Svetonio ( Aristotele e l'aristotelismo nella storia dell'estetica antica: origini, significato, svolgimento della Poetica», SIFC N. S. 2 (1921), p. 1-147 (p. 33). Secondo A. Primmer, “Akte und Spannung», p. 406 la tassonomia delle parti della commedia si originò nell'ambito dell'esegesi peripatetica a Menandro.

20 Il testo è citato secondo Aelii Donati quod fertur Commentum Terentii: Accedunt Eugraphi commentum et scholia bembina, recensuit P. Wessner, vol. I, Leipzig, 1902. 
Comoedia per quattruor partes dividitur, Prologum, Protasin, Epitasin, Catastrophen: est prologus velut praefatio quaedam fabulae, in quo solo licet praeter argumentum aliquid ad populum vel ex Poetae vel ex ipsius fabulae vel ex actoris commodo loqui; protasis primus est actus, initiumque dramatis; epitasis incrementum processusque turbarum, ut ita dixerim, nodus erroris; catastrophe, conversio rerum est ad jucundos exitus, patefacta cunctis cognitione gestorum.

La commedia si divide in quattro parti: prologus, protasis, epitasis, catastrophe: il prologus è una sorta di discorso preliminare al dramma vero e proprio, e solo lì è possibile, oltre a esporre l'argomento del dramma, dire qualcosa al popolo, concernente il poeta, o il dramma stesso, o l'attore; la protasis è il primo atto, l'inizio del dramma; l'epitasis è la parte nella quale l'intreccio si complica ed evolve - per così dire, il nodo dell'errore; la catastrophe è la parte in cui l'intreccio volge al lieto fine, dopo che tutti hanno acquisito la comprensione di ciò che è accaduto.

Analogamente, in de com. 7.1 si dice che il prologo cprecede il dramma propriamente detto» (antecedens veram fabulae compositionem). Nella definizione di 4,5 si nota come prologus sia l'unico nome con desinenza latina, e infatti in un passo precedente $(3,2)$ Evanzio precisa che «i Greci non hanno i prologhi alla nostra maniera, come li hanno i latini» (Graeci prologos non habent more nostrorum, quos Latini habent). L'erudito ha in mente i prologhi di Terenzio, nei

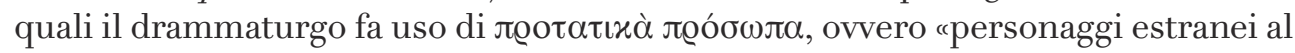
dramma» (personas extra argumentum accersitas); anche gli altri latini presentano a volte questi personaggi, ${ }^{21}$ mentre i Greci, dice Evanzio, non ne fanno uso.

Dalla lettura di questa tassonomia antica gli studiosi rinascimentali ricavavano un uso del termine prologo che era inconciliabile con quello aristotelico: se la definizione aristotelica di prologo come la parte del dramma che precedeva l'ingresso del coro implicava che esso fosse a tutti gli effetti parte dell'azione drammatica, Evanzio e Donato lo ponevano come una prefazione posta al di fuori di essa, recitata da un personaggio estraneo al dramma, ancora esente dalla regola dell'illusione drammatica. Ma Evanzio attribuiva queste caratteristiche al solo prologo della commedia latina, mentre Aristotele si riferiva alle tragedie e alle commedie greche: le peculiarità del prologo latino, in particolare la rottura dell'illusione drammatica, non potevano perciò trovarsi nella poesia drammatica greca. Collegando le classificazioni di Terenzio ed Evanzio alle categorie critiche degli scoli, alla definizione di prologo della Poetica aristotelica e all'impostazione retorico-oraziana della critica poetica, si giungeva a un rifiuto di qualsiasi soluzione drammaturgica che, nell'ambito della tragedia, portasse anche solo a far scricchiolare quella che oggi definiremmo la quarta parete del palcoscenico; 
erano dunque attive tutte le premesse per la censura teorica di una struttura drammaturgica così anomala quale il prologo euripideo.

\section{Il prologo euripideo secondo i moderni}

Va però detto che i pronunciamenti contro il prologo euripideo si fecero sentire solo relativamente tardi nella riflessione poetica cinquecentesca, a misura che i trattatisti acquisivano una maggiore consapevolezza critica e una maggiore risolutezza nell'esprimere giudizi di condanna sui modelli antichi, tra i quali era pur sempre incluso anche Euripide. Non si riscontra un siffatto atteggiamento, ad esempio, nei primi commenti alla Poetica aristotelica, il primo di Francesco Robortello (1548), il secondo di Vincenzo Maggi e Bartolomeo Lombardi (1550). Nel commentare il succitato passo della Poetica in cui si dà la definizione di prologo, Robortello prende anzi l'euripidea Ifigenia taurica euripidea ad esempio per illustrare la funzione del prologo tragico (Atque ut omnia notiora fiant exemplis appositis ex veterum poetarum tragoediis, proponam ego mihi haec consideranda in Iphigenia in Tauris Euripidis).${ }^{22}$ Non crea difficoltà a Robortello il fatto che il prologo di questa tragedia sia tra quelli che si presentano in forma più compiutamente diegetica. ${ }^{23}$

La vera e propria svolta in senso negativo nell'approccio critico verso il prologo euripideo si nota nella seconda metà del secolo, a partire dai Poetices libri septem di Giulio Cesare Scaligero (pubblicati postumi nel 1561). Egli osserva che Euripide «mescola in qualche misura il prologus con la protasis» (Sane Euripides cum protasi prologum fere miscet). ${ }^{24}$ Possiamo ritenere che con questa affermazione lo studioso intenda che le scene iniziali delle tragedie euripidee non sono ben integrate all'interno del dramma, in quanto sono affidate al monologo di un unico personaggio, ${ }^{25}$ che si comporta come se fosse slegato dalla fabula, quasi come un prologante della commedia latina.

22 Francisci Robortelli utinensis in librum Aristotelis de Arte Poetica explicationes, Florentiae, in officina Laurentii Torrentini, 1548, p. 119. La scelta dell'IT rispecchia del resto l'esemplarità di questa tragedia nella stessa Poetica aristotelica (cfr. 1454a 7, 1455a 18, 1455b 3), per cui cfr. E. Belfiore, “Aristotle and Iphigenia», in A. Oksenberg Rorty (a cura di), Essays on Aristotles' Poetics, Princeton, 1992, p. 359-377.

23 Sul prologo dell'IT cfr. Euripides Iphigenia in Tauris, ed. L.P.E. Parker, Oxford, 2016, p. 53-4. M. Duranti, “Iphigenia Taurica and the Narrative Artificiality of Euripides' Prologues", Skenè: Journal of Theatre and Drama Studies 3 (2) (2017), p. 151-71.

24 I. C. Scaliger, Poetices libri septem - Sieben Bücher über die Dichtkunst, Band I: Buch 1 und 2, ed. L. Deitz, Stuttgart, 1994, p. 180.

25 Riguardo al prologus latino, lo Scaligero nota che si tratta di una parte recitata da un unico personaggio (monoprosopos, J. C. SCALiger, Poetices libri septem, p. 154). 
Lo studioso aggiunge che per questa ibridazione Euripide è biasimato da alcuni critici (propter quod et a criticis damnatus est). A una ricerca su un ampio corpus di trattati rinascimentali, non risulta la presenza di questo giudizio critico su Euripide; lo Scaligero potrebbe però riferirsi agli scoli antichi, interpretando i loro appunti al tragediografo tramite le definizioni di Donato ed Evanzio. Come abbiamo visto nel paragrafo capitolo, l'indicazione più chiara sulla violazione dell'illusione drammatica in Euripide è in sch. Tro. 1 e Tro. 36. Tuttavia, questi scoli non erano disponibili a stampa, non essendo le Troades tra le tragedie i cui scoli furono pubblicati nel 1534 dal vescovo Arsenio Apostolio; bisognerebbe dunque supporre che lo Scaligero li abbia letti in forma manoscritta. Si può però anche ipotizzare che Scaligero abbia in mente lo scolio a Eur. Ph. 88, dove si dice che, con l'ingresso del servitore e di Antigone, l'andamento del dramma si

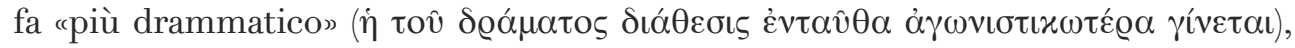
mentre prima, mentre le parole di Giocasta erano "trascinate in lungo", come se

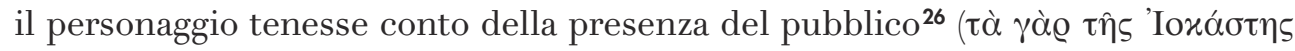

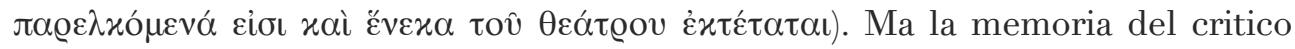
potrebbe anche andare al succitato sch. Aesch. Eum. 1a, dove si rimproverava a Euripide di violare l'illusione drammatica; gli scoli a Eschilo erano stati pubblicati nel 1552, sei anni prima della morte del nostro studioso (1558).

Se lo Scaligero non esprime ancora le riserve verso il prologo euripideo in prima persona, un altro celebre trattatista, Ludovico Castelvetro, mentre prima le parole una personale condanna di Euripide nel commento alla Poetica aristotelica (1571), in conformità con una dichiarata indipendenza di giudizio dagli autori antichi, incluso Aristotele. ${ }^{27}$ Castelvetro distingue tre tipologie di prologhi, unendo in questo termine il prologus di Evanzio e Donato e il prologos aristotelico. La prima modalità, tipica della commedia latina, csuole havere una persona seperata che è nominata prolago che fatto l'argomento della commedia non si vede più comparire, e questi appo Plauto è per lo più alcun dio e appo Terenzio è huomom. ${ }^{28}$

26 Lo scolio non dice che Giocasta parla direttamente agli spettatori, ma intende che il personaggio non ha un interlocutore interno al dramma al quale rivolgere il suo lungo discorso, e pertanto le sue parole suonano artificiose. Su questo punto R. NünLIst, The Ancient Critic, p. 342 n. 18, ha corretto R. MEIJERIng, Literary and Rhetorical Theories, p. 193-8. (cf. supra, n. 9)

27 Castelvetro dichiara di non voler non semplicemente commentare Aristotele, bensì elaborare una teoria poetica coerente. Pertanto, egli non esiterà a correggere e integrare le opinioni di Aristotele dove necessario (W. Romani (ed.), Lodovico Castelvetro, Poetica d’Aristotele vulgarizzata e sposta, Roma e Bari, 1978-9, vol. I p. 4).

28 W. Romani, Poetica, vol. I p. 141. Castelvetro biasima poco oltre Terenzio per essersi servito di prologanti umani, argomentando come non sia verosimile che un uomo 
La seconda modalità di prologo è tipica di Euripide, mentre la terza è adottata dagli altri tragediografi greci e da Aristofane: ${ }^{29}$

La seconda maniera de prolaghi non è del tutto seperata dall'azione come è del tutto seperata quella della comedia nuova trovata da latini, ma non è perciò congiunta come si converrebbe; e è quella che è usata da Euripide nelle sue tragedie nelle quali in su il principio introduce o dio o uomo a raccontare alcune o molte cose passate o presenti, per le quali altri intenda pienamente le cose seguenti; ma il più delle volte, anzi quasi sempre, con poca verisimilitudine, facendo che alcuno solo tenga un lungo ragionamento, e di cose la cui rammemorazione può essere stata fatta altra volta in tempo e in luogo più opportuno; ma questi cotale, introdotto a ragionare solo, non ragiona né del poeta, né delle cose lontane e separate dalla favola, né delle cose future che ragionevolmente non possa sapere, come fanno i prolaghi nelle commedie latine. La terza maniera di prolaghi è quella che è congiunta col rimanente della favola, e è parte, e parte principale e necessaria, della favola, e è legata per l'ordine delle cose col rimanente, non altramente che il capo è legato con l'altre membra del corpo; la quale Aristotele dice essere quella parte intera che è inanzi all'uscita del coro, e è molto commendata nelle tragedie di Sofocle e nelle comedie di Aristofane.

Come lo Scaligero, anche il Castelvetro colloca il prologo euripideo a metà tra il prologo separato della commedia latina e un regolare prologo tragico, sebbene non adotti la terminologia evanziana prologus-protasis, che pure ha certamente presente. Il prologo euripideo non può dirsi completamente distaccato in quanto, diversamente da quanto si verifica nei prologhi latini, il prologante rimane un personaggio del dramma e non si astrae da esso per introdurre elementi estranei all'intreccio come la lode del poeta o le polemiche con gli avversari; né rivela avvenimenti futuri che un personaggio umano non può conoscere. Il prologo euripideo non è però neppure congiunto pienamente con il dramma, nella misura in cui il prologante si produce in un lungo discorso, non plausibilmente motivato, pieno di dettagli che potrebbero essere rivelati in modo più graduale. Sulla base di questa idea di non integrazione del prologo euripideo nel dramma, ispirata dalla definizione evanziana del prologo separato alla latina e dal concetto di verosimiglianza drammatica, Castelvetro giunge alla conclusione che Aristotele non poteva avere inteso includere il prologo euripideo nella sue definizione di prologos come prima parte della tragedia, ma solo le scene iniziali di Eschilo, Sofocle, Aristofane. Vediamo qui pertanto le implicazioni ultime della forzata inclusione di Aristotele nell'ortodossia retorico-oraziana e nei suoi schemi di interpretazione. Che l'inclusione sia mediata anche attraverso la Retorica dello

conosca già l'esito di una vicenda che dovrebbe avvenire. Anche da questa valutazione emerge la centralità del principio della verosimiglianza. 
stesso Aristotele è provato, in relazione al prologo, dalla presenza di una citazione da quell'opera: per significare la piena inclusione dei prologhi di questi ultimi drammaturghi nel dramma, Castelvetro usa la similitudine della connessione della testa con il resto del corpo, con cui proprio nella Retorica si indica la connessione del proemio dell'orazione, appena paragonato al prologo tragico, con il resto $(1415 \mathrm{~b}$

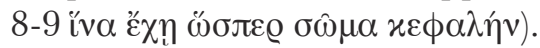

Nell'affermare che i prologhi di Sofocle e Aristofane, diversamente da quelli di Euripide, hanno ricevuto il plauso dei critici, Castelvetro sembra poi dimostrare la conoscenza dei commentatori antichi: è infatti negli scoli che si riscontra più volte la lode per i prologhi di Sofocle,$^{30}$ mentre nell'argomento delle Nuvole Aristofane è lodato per la scena iniziale («il prologo delle Nuvole è congegnato con

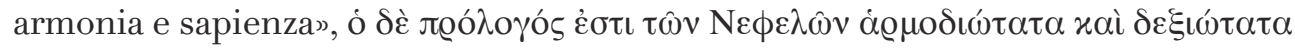

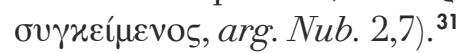

\section{Conclusioni}

La ricognizione condotta in questo articolo ha mostrato come dall'antichità alla prima età moderna si sia formato un quadro teorico sul prologo tragico, basato su una visione retorica della poesia e sulla nozione di verosimiglianza. Si è visto come la visione moderna del prologo derivi dalla lettura incrociata di testi antichi, riguardanti sia specificamente il prologo, come il De comoedia di Donato ed Evanzio, sia in generale l'arte drammatica, come l'Ars poetica di Orazio. Proprio la contaminazione dei testi antichi è all'origine di sorprendenti affermazioni come quella di Castelvetro, che esclude il prologo euripideo dalla categorizzazione della Poetica aristotelica. I teorici rinascimentali elaborarono così un insieme coerente di nozioni su come comporre e come valutare la poesia drammatica, destinato a far sentire a lungo la sua influenza. Per fare solo un esempio, quando il teorico del classicismo francese, l'abate d'Aubignac, afferma che, se si vuole dare un senso ragionevole alla definizione di prologo data da Aristotele, essa può essere riferita solo alle scene iniziali veramente integrate nell'azione drammatica, che non sono quelle tipiche di Euripide, ${ }^{32}$ ha di certo in mente le distinzioni del predecessore Castelvetro.

30 In proposito vd. MeiJering, Literary and Rhetorical Theories, p. 193.

31 Citato secondo D. Holwerda (ed.), Prolegomena de comoedia. Scholia in Acharnenses, Equites, Nubes (Scholia in Aristophanem 1.3.1.), Groningen, 1977.

32 “L'autre espèce de Prologue placé devant l'entrée du Chœur, contenait des choses qui non seulement concernaient le Sujet du Poème ; mai aussi qui lui étaient propres et incorporées, et qui en faisaient une véritable partie [...]: et si l'on veut réduire la définition d'Aristote à quelque sens raisonnable, on ne la peut entendre que de cette sorte de Prologue»; Авве́ d’Aubignac, La pratique du Théâtre, ed. H. Baby, Paris, 2001, p. 252. La pratique du theatre fu pubblicata nel 1657. 
Attraverso la mediazione dei trattatisti rinascimentali, le categorie critiche degli antichi hanno continuato ad essere normative per la poesia drammatica europea per secoli, imponendo rigidi confini al genere tragico. E così solo il Novecento ha saputo recuperare, per poi anche superarlo, lo spirito di sperimentalismo, sul filo della violazione delle consuetudini compositive, che era stato di Euripide.

\section{Marco Duranti}

Università degli Studi di Verona marco.duranti@univr.it 